\title{
TRATAMENTO CIRÚRGICO DA SAHOS
}

\author{
SURGERY FOR THE TREATMENT OF OSAHS
}

Daniel S Küpper ${ }^{1}$, Marcelo G J Leite ${ }^{1}$, Rodrigo L Nogueira ${ }^{1}$ Fabiana C P Valera ${ }^{1}$, José AA Oliveira²

\begin{abstract}
${ }^{1}$ Médico Assistente. Hospital das Clínicas da Faculdade de Medicina de Ribeirão Preto - USP. ${ }^{2}$ Docente. Divisão de Otorrinolaringologia. Departamento de Oftalmologia, Otorrinolaringologia e Cirurgia de Cabeça e Pescoço. Faculdade de Medicina de Ribeirão Preto - USP CorRESPONDÊNCIA: Daniel Salgado Küpper

Departamento de Oftalmologia, Otorrinolaringologia e Cirurgia de Cabeça e Pescoço.

Av. Bandeirantes 3900 - Monte Alegre. CEP 14049-900 - Ribeirão Preto - SP

e-mail: dskup@hotmail.com
\end{abstract}

Küpper DS, Leite MGJ, Nogueira RL, Valera FCP, Oliveira JAA. Tratamento cirúrgico da SAHOS. Medicina (Ribeirão Preto) 2006; 39 (2): 218-226.

RESUMO: O ronco deixou de ser apenas um incômodo social e junto com a Síndrome da Apnéia/Hipopnéia Obstrutiva do Sono (SAHOS) passou a ser considerado um problema de saúde. Segundo constatações atuais, o ronco e as apnéias estão associados a outras patologias, como: hipertensão arterial, acidente vascular cerebral, infarto do miocárdio e disfunção eréctil. $O$ tratamento ainda é um desafio para a medicina. $O$ tratamento atualmente considerado ideal é o uso de pressão positiva contínua nas vias aéreas (CPAP), porém diversas cirurgias vêm sendo propostas nos últimos anos como alternativas à resolução do problema. Neste capítulo iremos discutir as cirurgias comumente utilizadas para o controle do ronco e das apnéias.

Descritores: Síndromes da Apnéia do Sono. Ronco. LAUP. UPFP. Radiofreqüência.

\section{1- INTRODUÇÃO}

Até pouco tempo atrás, ronco era considerado um incômodo social, sem grande importância médica. A partir da década de 70 com a identificação da Síndrome da Apnéia Obstrutiva do Sono (SAHOS), o ronco passou a ser visto como uma patologia, por estar frequentemente associado a SAHOS. Com o passar do tempo e estudos mais específicos, passou-se a correlacionar a SAHOS com várias patologias como: hipertensão arterial, acidente vascular cerebral, infarto do miocárdio, disfunção sexual eréctil e até acidentes de trânsito, devido a sonolência excessiva, causada pela fragmentação do sono. ${ }^{1 / 5}$

Mesmo na ausência de apnéia, o ronco pode vir associado com sonolência diurna excessiva, perda subjetiva da capacidade de trabalho e cefaléia matinal. Mais ainda, é apontado como causa de brigas ou até separações matrimoniais. ${ }^{6,7}$
O ronco é originado da obstrução parcial à passagem do ar nas vias aéreas superiores, enquanto a apnéia surge da obstrução total do fluxo aéreo por um período maior que 10 segundos. Em ambas as situações a obstrução pode ocorrer em um nível isolado, ou em múltiplos níveis, como: cavidade nasal, nasofaringe, orofaringe ou hipofaringe, envolvendo estruturas como o palato mole, úvula, pilares amigdalianos, base de língua, músculos e mucosa da faringe, além da epiglote. ${ }^{8}$

O tratamento mais efetivo para a SAHOS de que se dispõe hoje em dia é o uso de pressão positiva contínua nas vias aéreas (CPAP). Porém além da aceitação dessa forma de tratamento não ser completa, é importante frisar que o CPAP não cura a patologia. Por isso muitos pacientes acabam procurando uma tentativa de resolução do problema através da cirurgia..,10

O tratamento cirúrgico da SAHOS permanece um desafio, não havendo um procedimento de esco- 
lha que, com certeza, promova resolução do fechamento das vias aéreas superiores (VAS) no sono. Até a introdução da uvulopalatofaringoplastia por Fujita em $1981^{11}$, as únicas opções cirúrgicas eram a amigdalectomia e a traqueostomia, esta última rejeitada por pacientes e médicos devido ao óbvio desconforto médico-social proporcionado pelo procedimento.

Nos últimos 25 anos vários procedimentos surgiram, na tentativa de solucionar a SAHOS como: uvulopalatofaringoplastia (UPFP), uvulopalatoplastia, avanço maxilo-mandibular, redução de base de língua, avanço de genioglosso, hioideopexia, isolados ou combinados entre si e com cirurgias nasais.

Os resultados destas várias modalidades permanecem controversos. Na opinião dos autores as cirurgias apresentam bons resultados, quando bem indicadas. Por isso achamos importante uma boa definição do sítio obstrutivo, antes da indicação de qualquer técnica.

Além de um bom exame otorrinolaringológico, a manobra de Müller se mostra um exame importante. Apesar da maior parte dos estudos mostrarem que esta manobra não tem um bom valor preditivo para avaliar adequadamente o sítio de obstrução ${ }^{12,13}$, ela vem sendo utilizada na tentativa de auxiliar na obtenção de sucesso cirúrgico. Por ser realizada com o paciente acordado a manobra de Müller, talvez falhe em indicar com exatidão o sítio de obstrução. Dessa forma Croft e Pringle ${ }^{14}$ propuseram a realização da mesma manobra com o paciente dormindo após injeção intravenosa de um indutor de sono. Isto tem-se mostrado mais preciso na identificação do locus da obstrução das vias aéreas superiores, porém ainda carece de estudos randomizados para validá-lo. Por outro lado fica a dúvida sobre se o sono induzido por droga produziria o mesmo dano que o sono normal.

A seguir apresentamos as cirurgias que usualmente realizamos, com suas respectivas indicações, contra-indicações, resultados e complicações.

Apesar das controvérsias acerca da obesidade na gênese e manutenção do ronco e da SAHOS, para melhor resultado cirúrgico optamos por trabalhar com indivíduos com um IMC máximo de $30 \mathrm{Kg} / \mathrm{m}^{2}$.

\section{2-UVULOPALATOFARINGOPLASTIA (UPFP)}

Técnica de Fairbanks modificada

\section{1- Histórico}

Ikematsu em 1952 propôs o primeiro procedimento cirúrgico para tratamento do ronco. Essa técni- ca foi modificada por diversos autores até 1981, quando Fujita et al. ${ }^{11}$ introduziram uma cirurgia para o tratamento do ronco e apnéia, visando aumentar o espaço aéreo na orofaringe dos pacientes com SAHOS, denominando-a de uvulopalatofaringoplastia. Posteriormente, Fairbanks realizou modificações objetivando maximizar a lateralização dos pilares posteriores, preservar a ação de esfíncter da musculatura palatonasofaríngea e diminuir a retração cicatricial do palato. A técnica de Fairbanks persiste como a técnica atualmente mais utilizada para a uvulopalatofaringoplastia. ${ }^{15,16,17}$

\section{2- Indicação}

Atualmente a uvulopalatofaringoplastia é o procedimento cirúrgico mais comumente utilizado para o tratamento do ronco e apnéia. ${ }^{18}$

É um procedimento para pacientes com obstrução localizada na região da orofaringe (Fujita et al. ${ }^{11}$ ). Os achados no exame físico são: palato mole alongado, paredes laterais de faringe redundantes, úvula longa e volumosa, tonsilas palatinas aumentadas (graus II, III e IV). Melhores resultados são alcançados em pacientes sem alterações crânio-faciais e com índice de massa corporal (IMC) inferior a $30 \mathrm{Kg} / \mathrm{m}^{2}, 15,18$

Pacientes com esses achados e que tenham diagnóstico de ronco primário ou Síndrome da Apnéia/ Hipopnéia Obstrutiva do Sono de grau leve a moderado podem ser beneficiados com esse tipo de abordagem cirúrgica.

Deve ser indicada com cuidado, pois suas falhas estão diretamente relacionadas com indicações errôneas em pacientes com obstrução de vias aéreas em níveis mais baixos. ${ }^{18}$

\section{3- Técnica}

O objetivo da uvulopalatofaringoplastia é promover uma abertura da orofaringe. Para isso são removidos as tonsilas palatinas e o excesso do palato, com a construção de uma neo-úvula e abertura lateral dos pilares amigdaleanos.

A cirurgia é feita com o uso de anestesia geral, sob intubação oral. O paciente é colocado em decúbito dorsal, com leve extensão da cabeça (posição de Rosen). A cavidade oral é exposta com uso do abridor de boca.

Inicialmente é feita a remoção das tonsilas palatinas por dissecção, preservando ao máximo a mucosa dos pilares amigdaleanos. Realiza-se então uma cuidadosa hemostasia das lojas amigdaleanas através de pontos com fio categute 3-0 (Figura 1), evitando-se o uso de cautério para este passo. ${ }^{15}$ 


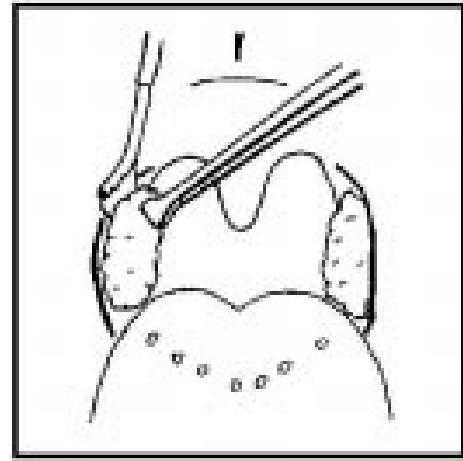

Figura 1: Antes da realização da UPFP é feita a remoção das tonsilas palatinas por dissecção.

A seguir, realiza-se incisão no palato mole com tesoura, lateralmente à úvula, na junção dos pilares amigdalianos dos dois lados (Figura 2). Os pilares são suturados provocando um fechamento dos $2 / 3$ inferiores das lojas amigdalianas, sem que haja uma tensão excessiva na sutura. (Figura 3 ).

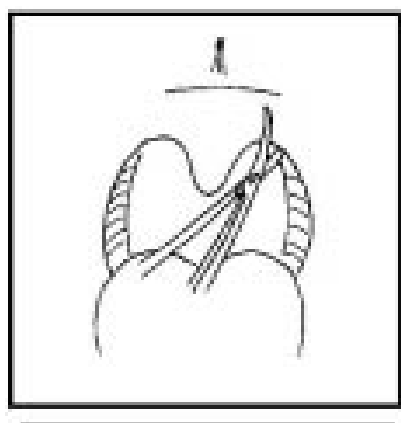

Figura 2: O primeiro passo na UPFP é a incisão no pálato mole com tesoura lateralmente à úvula

Avalia-se a porção em excesso do palato mole e úvula, pela manobra digital de comprimir o palato mole até encontrar a parede posterior da faringe e assim determinar o limite da ressecção. A incisão na porção anterior da mucosa com bisturi lâmina 11 é feita a partir desse ponto em direção a borda livre do palato. Segue-se com a dissecção do subcutâneo e musculatura do palato, utilizando-se de um descoladoraspirador, deixando a porção posterior da mucosa palatal $1 \mathrm{~cm}$ maior do que a porção anterior e confeccionando-se uma neo-úvula. (Figura 4) ) $^{15 / 20}$.

As incisões laterais do palato são suturadas com fio vicryl 30, tracionando-se lateralmente o arco palatino, sem deixar área cruenta, unindo-se a mucosa posterior à anterior. (Figura 5) 15,18,19,20 $^{\text {. }}$

A antibioticoterapia profilática é feita no início do procedimento com uso de Cefazolina endoveno$\mathrm{sa}^{15,18,19}$.

\section{4- Contra-indicações}

Pacientes com sítio de obstrução que não seja a orofaringe (ex: macroglossia), devem ser avaliados para o uso de outro tipo de técnica cirúrgica ou outra modalidade de tratamento.

Pacientes com malformações esqueléticas faciais (micrognatia) ou outras deformidades craniofaciais são contra indicados para a UPFP.

Classificações de apnéia pela polissonografia como de grau grave ou como apnéias centrais devem ser tratadas com CPAP e não com esta modalidade cirúrgica. Outra opção para alguns pacientes com apnéia de grau grave é a cirurgia de Avanço Maxilomandibular. ${ }^{15}$

Portadores de doenças sistêmicas graves ou descompensadas (exs: discrasias sangüíneas, cardiopatias, distúrbios metabólicos) tem contra-indicação relativa. Podem ser operados desde que a patologia de base esteja compensada ou não os coloque em risco de vida durante um procedimento cirúrgico. ${ }^{15,18}$

\section{5- Resolubilidade}

Os resultados da UPFP são muito variáveis na literatura. Trabalhos diferentes mostram índice de resolução que variam de $20 \%$ a $80 \%$. Acredita-se que essa diferença ocorra devido a uma falta de padronização na indicação da cirurgia. Geralmente decorre de uma avaliação pré-operatória falha, onde não foi localizado o sítio correto de obstrução. Portanto, a correção apenas da região de orofaringe não trará benefícios se a obstrução está em outro local. ${ }^{15}$

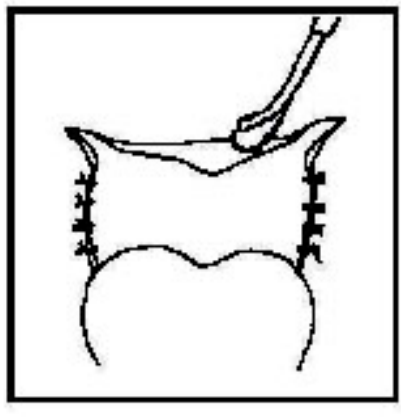

Figura 4: Procede-se a dissecção do subcutâneo e musculatura do pálato e confecção de uma neo-úvula.

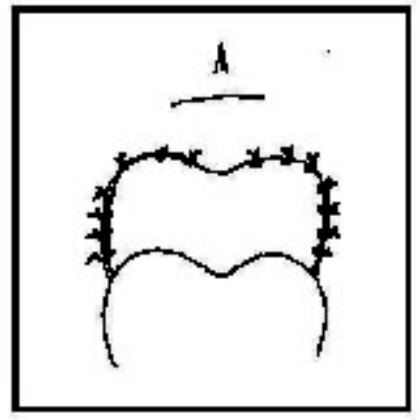

Figura 5: As incisões laterais do pálato são suturadas sem deixar área cruenta. 
Estudos atuais mostram $80 \%$ de sucesso cirúrgico nos primeiros meses. Tomita, S, no ano de 2000, concluiu que a taxa de sucesso em curto prazo foi de $96,2 \%$. Porém, após 12 meses, essa taxa caiu para $78,6 \%$. Não houve casos de piora do ronco no pósoperatório. ${ }^{17,21}$

\section{6- Complicações}

A morbidade e mortalidade pós-operatória na uvulopalatofaringoplastia (UPFP) são pouco freqüentes e devem ser minimizadas com uma avaliação préoperatória completa (polissonografia, historia clinica e exame físico detalhados). ${ }^{20}$

Complicações relacionadas a UPFP podem ser divididas em precoce (intra-operatórias e pós-operatórios imediatos) ou tardia. Normalmente estão relacionadas a técnicas e procedimentos inadequados. ${ }^{15}$

\section{- Precoce}

As complicações mais comuns no pós-operatório imediato são respiratórias, incluindo obstrução de vias aéreas e hipoxia (75\% das complicações intraoperatórias das uvulopalatofaringoplastia). Edema das vias aéreas pode levar o paciente a uma piora da apnéia no pós-operatório imediato. Flacidez e colapso das vias aéreas, dificuldade de intubação ou hemorragias pós-operatórias também podem contribuir para essa piora. A equipe deve estar preparada para o uso de CPAP em ambiente de CTI. ${ }^{20}$

Sedação pré-operatória deve ser feita com muito cuidado pois o uso de relaxantes e anestésicos pode promover um maior relaxamento da musculatura das vias aéreas. O uso de narcóticos, com o Fentanyl, é o maior fator de risco para apnéia pós-extubação. ${ }^{15}$

Anormalidades anatômicas encontradas em pacientes apnéicos podem dificultar a intubação. Técnicas alternativas de intubação podem ser necessárias (retrógrada, endoscópica ou nasal) e toda a equipe deve estar preparada para a realização de traqueostomia de urgência.

Insuficiência velo-faríngea pode ocorrer em $20 \%$ dos pacientes submetidos a UPPP. Na maioria das vezes é transitória, mas caso persista por mais de 2 meses, a correção cirúrgica pode ser necessária. Nesses casos provavelmente o tecido palatal foi removido em excesso. ${ }^{15,20}$

Deiscência da sutura da parede lateral da faringe pode levar a um sucesso parcial da cirurgia. Infecção cirúrgica pode ser evitada com o uso de antibioticoterapia.
Dor é uma queixa freqüente e aparece quando cessa o efeito da anestesia. Normalmente piora até o terceiro dia de pós-operatório, quando atinge seu pico de intensidade. Deve ser controlada com analgésicos potentes, lembrando que os anestésicos centrais podem aumentar as apnéias e, portanto, o paciente deve ser monitorado com atenção. $\mathrm{O}$ uso de anestésicos tópicos também é benéfico. O paciente com dor deixa de se alimentar e ingerir líquidos, ficando com as mucosas desidratadas e piorando a dor. Hidratação deve ser estimulada pela equipe médica. ${ }^{15}$

\section{- Tardia}

A amputação da úvula pode gerar sensação de boca seca, corpo estranho na faringe e dificuldade de gargarejo. ${ }^{15,18}$

O uso prolongado de abridor de boca durante cirurgias mais demoradas pode levar o paciente a desenvolver distúrbios do paladar. Acredita-se que a pressão excessiva sobre a base da língua lese terminações nervosas. O controle da força do abridor e do tempo cirúrgico minimiza esse problema. ${ }^{15}$

Estenose nasofaríngea é a complicação mais temida da UPFP. Em geral está relacionada à inabilidade do cirurgião. Fatores de risco para essa complicação são: infecção, uso em excesso de eletrocautério, laceração excessiva da mucosa, necrose e ressecção exagerada dos pilares posteriores. Apesar de ser rara representa uma consequiência grave em virtude da limitação da correção e pelo seu caráter recidivo. ${ }^{15,18,20}$

\section{3- UVULOPALATOPLASTIA (UPP)}

A primeira descrição cirúrgica data de 1952, quando Ikematsu observou que uma paciente reclamava de ronco excessivo e apresentava úvula longa com "flap" palatal posterior também alongado. Após a cirurgia, onde removeu parte da úvula e o palato redundante a paciente apresentou melhora total. ${ }^{15}$

A técnica inicial de Ikematsu foi aperfeiçoada, fazendo com que a parede lateral também fosse reparada, dando origem a uvulopalatofaringoplastia. Fujita aperfeiçou esta última técnica e popularizou-a na década de $1981 .^{11}$

A Uvulopalatofaringoplastia mostrou ser, em alguns casos, uma cirurgia efetiva para ronco e SAHOS, porém apresenta algumas complicações temíveis, como: estenose faríngea, insuficiênncia velofaríngea, sangramentos, edema importante com insuficiência-respiratória aguda. Além da necessidade de 
uso de anestesia geral e internação hospitalar, o que encarece o seu custo. ${ }^{11,15 / 21}$

$\mathrm{Na}$ tentativa de minimizar as complicações e custos, mantendo a eficácia dos resultados do procedimento, Kamani em 1990 desenvolveu a técnica de uvulopalatoplastia a Laser (LAUP - Laser-Assisted Uvulopalatoplasty), que passou a ser utilizada em larga escala. ${ }^{22}$

O grande benefício trazido ao paciente por esta técnica, é que passou a ser realizada ambulatorialmente e com anestesia local.

\section{1- Técnica}

Inicia-se a anestesia com spray de lidocaína a $10 \%$ em toda a região palatal, úvula e base de língua (Figura 6). Seguida por infiltração de solução de lidocaína a $2 \%$ e adrenalina a 1:200000 em 3 pontos: base de úvula e região lateral de palato mole, próximo aos pilares amigdalianos. ${ }^{22}$

O procedimento é realizado com laser de $\mathrm{CO} 2$ (Figura 7), utilizandose uma peça denominada“" back-stop", que protege a

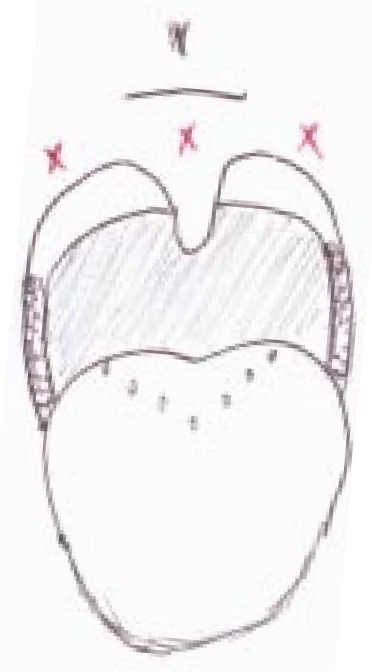

Figura 6: Infiltração anestésica. parede posterior da faringe da ação do laser. A potência do laser é regulado para 15 a $20 \mathrm{~W}$ em modo contínuo, e duas incisões são realizadas, laterais a base da úvula, verticais (com leve inclinação lateral), abrangendo todo o "flap" palatal posterior que se encontra em excesso. É necessário respeitar o pilar anterior de modo a não lesar os músculos tensor e levantador do véu palatino, a fim de evitar complicações. ${ }^{22}$

Após as incisões verticais procede-se a vaporização da úvula (Figura 8), utilizando-se o swiftlase®, que faz com que a luz do laser seja desfocada e desse modo a lesão tecidual ocorra superficialmente. Reduz-se a úvula a cerca de 60 a $90 \%$ do inicial ${ }^{22}$

A uvulopalatoplastia pode ser realizada com bisturi de alta-frequência Apesar de poucos estudos terem sido realizados com este aparelho, na prática no nosso ambulatório o mesmo tem-se mostrado eficaz, com índice de complicações e sucesso semelhantes a LAUP. ${ }^{22}$

O ponto favorável da utilização do cautério em vez do laser é o custo do equipamento, que chega a ser 30 vezes menor. A técnica de anestesia é semelhante a descrita para a LAUP, regulando-se o aparelho para $80 \%$ corte e $20 \%$ coagulação para as incisões laterais e $80 \%$ coagulação e $20 \%$ corte para a vaporização da úvula. ${ }^{22}$

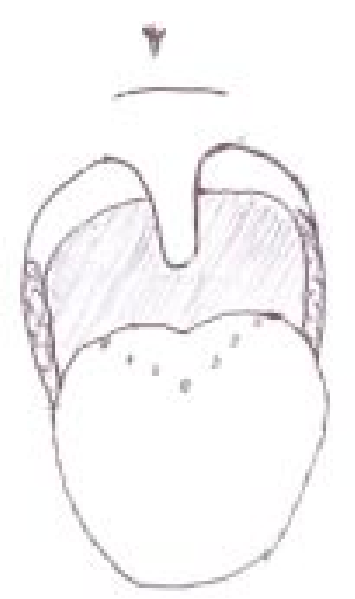

A

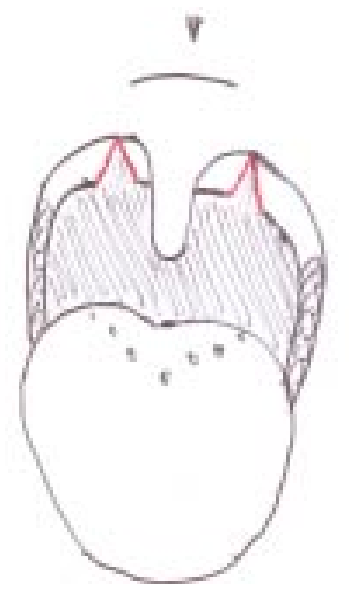

B

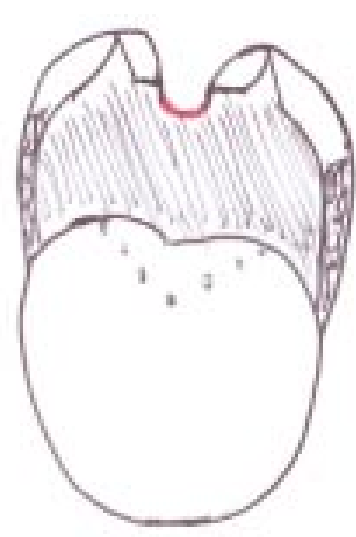

C

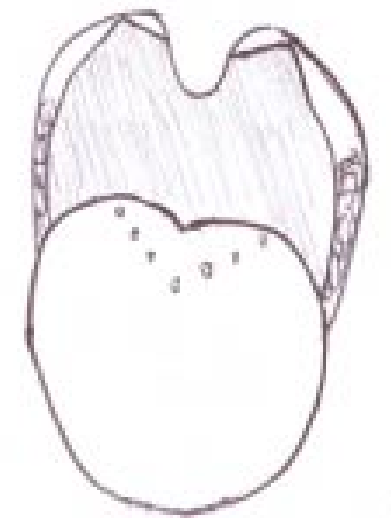

D

Figura 8: Visão da cavidade orofaríngea. A: Após infiltração - visão pré-operatória. B: Incisão vertical em pilares posteriores. C: Cauterização da úvula. D: Pós-operatório: retração esperada de pilar posterior e pálato mole. 


\section{2-Indicações, contra-indicações e resolutibilidade}

A uvulopalatoplastia tem-se mostrado bastante eficaz para a correção de ronco primário, com índice de sucesso a curto prazo ( 3 meses ) de cerca de $75 \%$ a $92 \%{ }^{1 / 6}$ e a longo prazo ( superior a 2 anos) de cerca de $62 \%$ a $74 \%$ de resolução. ${ }^{22 / 28}$

Porém quando a proposta da utilização da UPP é para o tratamento da SAHOS os trabalhos existentes ainda são bastante controversos. Enquanto alguns autores relatam melhora dos índices de distúrbios respiratórios pós-cirurgia, outros mostram resultados desfavoráveis e em alguns casos, houve piora da SAHOS após o procedimento. ${ }^{22 / 38}$

A opinião de muitos autores é de que a UPP não se mostra eficaz para casos de apnéia grave e moderada, porém tem indicação para casos de apnéia leve. ${ }^{22 / 33}$ Os trabalhos atuais são conflitantes quanto aos resultados em pacientes com apnéia mesmo leve $\mathrm{e}^{34 /}$ 40. A Academia Americana de Distúrbios de Sono contra-indica o procedimento para o tratamento de SAHOS, e só o recomenda para os casos de ronco primário $^{33}$. Levantamento recente, onde os dados de mais de 300 trabalhos acerca da LAUP foram analisados, mostrou que nenhum pode ser considerado conclusivo, em decorrência de erro na metodologia e análise dos dados. ${ }^{34}$

As contra-indicações da UPP: apnéia grave e moderada, hipertensão arterial descontrolada, pouca abertura bucal, fenda palatal, insuficiência velo-faríngea, e em pacientes não cooperativos ou com reflexo nauseoso intenso. ${ }^{22 / 28}$

A opinião dos autores é que a UPP pode ser indicada em casos de roncopatia primária e em alguns casos selecionados de SAHOS leve. Pacientes com IMC < 26, IDR < 10, sem hipertrofia de base de língua, com "flap" palatal posterior grande (cerca de $1 \mathrm{~cm}$ ), e ausência de amigdalas palatinas, podem ser submetidos a UPP com boas chances de sucesso.

As complicações mais freqüentes, observadas são: sangramento, normalmente leve e quando presente, facilmente corrigido com cautério. A dor é o maior efeito colateral desta técnica, podendo ser de moderada a severa. Inicia ao término da anestesia, atinge o pico em 3-5 dias e dura até 10 dias, sendo necessário, muitas vezes, o uso de analgésicos potentes. Reação vaso-vagal na injeção de anestésico não é freqüente e pode ser decorrente da administração do anestésico em algum vaso. Insuficiência velo-faríngea transitória pode ocorrer nos primeiros dias; sua permanência pode significar lesão da musculatura palatal e fonoterapia pode ser tentado para resolução. Raramente pode ocorrer estenose faríngea, complicação temerária e de difícil resolução, que leva a piora do ronco e das apnéias. ${ }^{22 / 38}$ Esta piora se deve a retração cicatricial leve que mesmo quando, não suficiente para causar estenose, pode levar a diminuição da luz faríngea. ${ }^{35}$

\section{4- CIRURGIAS NASAIS}

A obstrução nasal é um problema relativamente comum podendo comprometer a qualidade de vida e, principalmente agravar quadros de apnéia do sono. As principais causas de obstrução são: desvio de septo nasal, hipertrofia de conchas nasais, pólipos nasais, lesões traumáticas, colapso de válvula nasal, entre outros. $^{41}$

A correção das alterações estruturais nasais ainda é um assunto controverso. Apesar de alguns autores afirmarem que a correção cirúrgica do nariz tem efeito limitado no tratamento da SAHOS exitem vários argumentos a favor desta conduta. ${ }^{42,43,44}$

Outro aspecto relevante é que muitos pacientes ao longo da evolução da SAHOS, mesmo tendo realizado a cirurgia, necessitarão fazer uso do CPAP, e a cirurgia nasal será fundamental para aumentar a tolerância ao aparelho.

\section{5- SEPTOPLASTIA}

A correção do desvio de septo nasal tem o objetivo de equilibrar o fluxo nasal de maneira simétrica em ambas as fossas nasais. O septo nasal tem um papel importante tanto na função quanto na estética devendo o cirurgião detectar a área responsável pela obstrução visando realizar uma cirurgia mais eficiente e conservadora.

Ao longo das décadas as técnicas cirúrgicas foram se modificando. No século XIX a correção do desvio baseava-se na remoção total do septo. Na primeira década do século XX alguns cirurgiões como Freer (1902) passaram a realizar uma ressecção submucosa, mais conservadora. Com a popularização das cirurgias mais conservadoras muitos outros cirurgiões se destacaram como Killian (1904),Metzenbaum (1929), Peer (1937) e Cottle (1950) ${ }^{45,46}$. 


\section{6- CIRURGIA DAS CONCHAS NASAIS}

Ao longo dos anos observou-se que a cirurgia isolada do septo nasal, em alguns casos, não melhorava os sintomas obstrutivos. Assim a partir dos estudos de Holmes em 1900 as conchas nasais passaram a ser estudadas e novas técnicas foram desenvolvidas.

Em 1908 Escart sistematizou a técnica de turbinectomia com tesoura com o objetivo de melhorar o fluxo nasal e facilitar a drenagem do seio maxilar. Quando a hipertrofia é identificada como causa da obstrução, ela deve ser tratada ${ }^{47}$.

Nos dias atuais a hipertrofia das conchas pode ser tratadas de inúmeras maneiras destacando-se: infiltração nasal, luxação e fratura, cauterização com bisturi elétrico ou radiofrequiência, vaporização a lazer, turbinoplastia ou turbinectomia.

\section{7- OSTEOTOMIA MANDIBULAR COM AVANÇO DO GENIOGLOSSO}

Proposta por Riley et al. em 1984 esta cirurgia foi utilizada primeiramente com o objetivo de abranger as vias aéreas superiores como um todo abordando diretamente a velofaringe por UPFP e indiretamente a hipofaringe por osteotomia mandibular com avanço do músculo genioglosso associado a suspensão do osso hióide, tracionando anteriormente a musculatura aderida ao osso hióde e o músculo genioglosso inserido no mento. Desta forma diminui-se a colapsibilidade as hipofaringe durante o sono ${ }^{48}$.

\section{8- GLOSSECTOMIA PARCIAL}

\section{1- Histórico}

A glossectomia parcial permite diminuição do volume da base da língua posteriormente em relação a orofaringe como também alteração do volume laterolateral, pela remoção parcial da base da língua que pode ser na região central ou lateral.

Alguns cirurgiões associam a UPFP a glossectomia parcial no mesmo procedimento. Miljeteg foi um dos pioneiros e obteve $90 \%$ de satisfação subjetiva dos pacientes tratados e na polissonografia $67 \%$ apresentaram resposta satisfatória ao tratamento. Outra possibilidade de ressecção da base da língua por acesso cervical foi proposta por Chabolle, porém por ser muito invasivo esta técnica foi abandonada ${ }^{49}$.

\section{2- Radiofrequência de Base de Língua}

\section{- Técnica}

Correntes de radiofreqüência tem sido utilizadas em aparelhos cirúrgicos para realização de glossectomias. O gerador de energia desenvolvido pela empresa americana Somnus ® (daí o nome do procedimento ser divulgado como Somnoplastia) utiliza energia de radiofreqüência, tendo como objetivo a destruição celular por termólise, sem cauterizar ou queimar o tecido. A corrente que circula pelo eletrodo gera um campo magnético que produz calor elevando a temperatura levando a uma desnaturação protéica com consequiente morte celular e posterior fibrose local $^{50}$.

O uso da radiofrequiência na prática otorrinolaringológica iniciou-se em 1997, quando o FDA reconheceu sua aplicação no palato mole e úvula para o tratamento do ronco primário, e posteriormente, em 1998, para apnéia obstrutiva de grau leve. Em casos de apnéia grave seu uso foi liberado para aplicação em base de língua e cornetos nasais.

\section{- Resolubilidade}

A aplicação da radiofrequiência em base de língua demonstrou ser eficaz na redução do volume da língua em 17\% em média com conseqüente ampliação da via aérea ${ }^{49}$, em pacientes com SAHOS.

\section{- Complicações}

As complicações mais temidas deste procedimento são o edema da base da língua com conseqüente obstrução da via aérea que pode ser letal no pós operatório imediato ou nas primeiras 24-48 horas. Alguns autores preconizam a traqueotomia prévia como forma de evitar estas complicações.

\section{9- TRAQUEOSTOMIA}

A traqueostomia foi o principal e melhor tratamento para a SAHOS na década de 70 em uma época que a maioria dos pacientes diagnosticados apresentavam obesidade mórbida ou SAHOS grave. Até a introdução do CPAP e da UPFP na década de 80 a traqueostomia era a única alternativa. Atualmente ela é utilizada esporadicamente, geralmente como uma solução temporária. A traqueotomia terapêutica está indicada em casos de SAHOS grave associada a intensa dessaturação e arritmia cardíaca em que o CPAP não foi tolerado e o BiPAP associado a oxigenoterapia não resolveu. A permeabilização das VAS reduz a mortalidade dos pacientes com SAHOS. 
Küpper DS, Leite MGJ, Nogueira RL, Valera FCP, Oliveira JAA. Surgery for the treatment of OSAHS. Medicina (Ribeirão Preto) 2006; 39 (2): 218-226.

ABSTRACT: Nowadays, snoring is not only a social problem, but it became a disease, especially when associated to obstructive sleep apnea and hypopnea syndrome (OSAHS). It is associated to many other illnesses, such as systemic arterial hypertension, stroke, myocardial infarction and erectile dysfunction. The treatment for snoring and OSAHS remains a challenge for medicine. Although the gold standard treatment is CPAP, many surgeries have been proposed as alternatives to its resolution. In this chapter, the authors will discuss the surgical procedures most commonly performed for snoring and OSAHS control.

Keywords: Sleep Apnea Syndromes. Snoring. LAUP. UPPP. Radiofrequency.

\section{REFERÊNCIAS}

1 - Guilleminault C, Connoly S, Winkle. Cardiac arrythmia and conduction disturbance during sleep in 400 patients with sleep apnea syndrome. Am J Cardiol 1983; 52: 490-4.

2 - Findley LJ, Barth JT, Powers DC, Wilhoit SC, Boyd DG, Suratt PM. Cognitive impairment in patients with obstructive sllep apnea and associated hypoxemia. Chest 1986; 90: 686-90.

3 - Guilleminault C. Natural history, cardiac impact and long-term follow-up of sllep apnea syndrome. In : Guilleminault C, Lugaressi $E$, eds. Long-term evolution and natural history of sllep disorders. New York: Raven Press; 1983; p107-25.

4 - Partinen M, Jamieson A, Guilleminault C. Long-term outcome foro obstructive sllep apnea syndrome patients: Mortality. Chest 1988; 94: 1200-4.

5 - Findley LJ, Unverzagt ME, Suratt PM. Automobile accidents involving patients with obstructive sleep apnea. Am Rev Respir Dis 1988; 138: 337-40.

6 - Hoffstein V, Mateika S, Nash S. Comparing percepetions and measurements of snoring. Sleep 1996; 19: 783-9.

7 - Lindberg E, Taube A, Janson C, Gislason T, Svardsudd K, Boman G. A 10-year follow-up of snoring in men. Chest $1998 ; 114: 1048-55$

8 - Thorpy M. American Sleep Disosdres Association Report: Practice parameters for the treatment of obstructive sleep apnea in adults: the efficacy of surgical modifications of the upper airway. Sleep 1996; 19:152-5.

9 - Krieger J. Long-term compliance with nasal continuous positive airway pressure (CPAP) in obstructive sleep apnoea patient and nonapneic snorers. Sleep 1992; 15 (Suppl 6): 42-6.

10 - Collop NA, Block AJ, Hellard D. The effect of nightly nasal CPAP tratment on underlying obstructive sleep apnea and pharyngeal size. Chest 1991; 99: 855-60.

11 - Fujita S, Conway W, Zorick F, Roth T. Surgical correction of anatomic abnormalities in obstructive sleep apnea syndrome:
Uvulopalatopharyngoplasty. Otolaryngol Head Neck Surg 1981;89:923-34.

12 - Katsantonis GP, Maas CS, Walsh JK. The predictive efficacy of the Muller maneuver in uvulopalatopharyngoplasty. Laryngoscope 1989; 99:677-80.

13 - Petri N, Suadicani P, Wildschiodtz G, Bjorn-Jorgensen J. Predictive value of Muller maneuver, cephalometry and clinical features for the outcome of uvulopalatopharyngoplasty. Evalution of predictive factors using discriminant analysis in 30 sleep apnea patients. Acta Otolaryngol 1994; 114:565-71.

14 - Croft CB, Pringle M. Sleep nasendoscopy: a technique of assessment in snoring and obstructive sleep apnoea. Clin Otolaryngol 1991; 16:504-9.

15 - Pinto JA; Freitas MLAS. Uvulopalatofaringoplastia In: Pinto JÁ. Ronco e apnéia do sono. Rio de Janeiro: Revinter, 2000 p151-60.

16 - Fairbanks DN. Uvulopalatopharyngoplasty complications and avoidance strategies. Otolaryngol Head Neck Surg 1990; 102:239-45.

17 - Tomita S, Abreu HF. Avaliação de novo método de uvulopalatofaringoplastia no tratamento do ronco. Arq da Fund Otorrinolaringol 2000; 4: 11-6.

18 - Zonato Al; Gregório LC. Tratamento cirúrgico da síndrome da apneia e hipopnéia obstrutiva do sono. In: Sociedade Brasileira de Otorrinolaringologia Tratado de otorrinolaringologia. São Paulo: Roca; 2003. p. 329-48.

19 - Fairbanks DNF. Uvulopalatopharyngoplasty: Method of Fairbanks In: Fairbanks DNF, Fujita S. Soring and obstructive sleep apnea. $2^{\text {th }}$ ed, New York: Raven Press, 1994, cap7-D, p118-23.

20 - Kim JA, Lee JJ, Jung HH. Predictive factors of immediate postoperative complications after uvulopalatopharyngoplasty. Laryngoscope 2005; 115:1837-40.

21 - Ray RM, Bower CM. Pediatric obstructive sleep apnea: the year in review. Curr Opin Otolaryngol Head Neck Surg 2005; 13:360-5. 
22 - Kamani YV. Laser $\mathrm{CO}_{2}$ for snoring: preliminary results. Acta Otorhinolaryngol Belg 1990; 44:451-6.

23 - Carenfelt C. Laser uvulopalatoplasty in tratment of habitual snoring. Ann Otol Rhino Laryngol 1991; 100:451-4.

24 - Mickelson SA. Laser-assisted uvulopalatoplasty for obstructive sleep apnea. Laryngoscope 1996; 106:10-3.

25 - Krespi YP, Pearlman SJ, Keidar A. Laser-assisted uvulopalatoplasty for snoring. J Otolaryngol 1994; 23:32834.

26 - Kamani YV. Outpatient treatment of snoring with CO2 laser: laser-assisted UPPP. J Otolaryngol 1994; 23:391-4.

27 - Walker RP, Grigg-Damberger MM, Gopalsami C, Totten MC. Laser assisted uvulopalatoplasty for snoring and obstructive sleep apnea: results in 170 patients. Laryngoscope 1995; 105:938-43.

28 - Walker RP, Gatti WM, Poirier N, Davis JS. Objective assessment of snoring before and after laser-assisted uvulopalatoplasty. Laryngoscope 1996; 106:1372-.7.

29 - Osman EZ, Osborne JE, Hill PD, Lee BW, Hammad Z. Uvulopalatopharyngoplasty versus laser assisted uvulopalatoplasty for the treatment of snoring: An objective randomised clinical trial. Clin Otolaryngol Allied Sci 2000; 25:305-10.

30 - Mickelson SA, Ahuja A. Short-term objective and long-term subjective results of laser-assisted uvulopalatoplasty for obstructive sleep apnea. Laryngoscope 1999; 109:362-7.

31 - Walker RP, Garrity T, Gopalsami C. Early polysomnographic findings and long-term subjective results in sleep apnea patients treated with laser-assisted uvulopalatoplasty. Laryngoscope 1999; 109:1438-41.

32 - Seemann RP, DiToppa JC, Holm MA, Hanson J. Does laserassisted uvulopalatoplasty work? An objective analysis using pre and postoperative polysomnographic studies. J Otolaryngol 2001; 30:212-5.

33 - Kyrmizakis DE, Chimona TS, Papadakis CE, Bizakis JG, Velegrakis GA, Schiza, Siafakas NM, Helidonis ES. Laserassisted uvulopalatoplasty for the treatment of snoring and mild obstrutictive sleep apnea syndrome. J Otolaryngol 2003; 32:174-9.

34 - Ryan CF, Love LL. Unpredictable results of laser assisted uvulopalatoplasty in the treatment of obstructive sleep apnoea. Thorax 2000; 55:399-404

35 - Finkelstein Y, Stein G, Ophir D, Berger R, Berger G. Laserassisted uvulopalatoplasty for the management of obstructive sleep apnea: myths and facts. Arch Otolaryngol Head Neck Surg 2002; 128:429-34.

36 - Fergunson KA, Heighway K, Ruby RR. A randomized trial of laser-assisted uvulopalatoplasty in the treatment of mild obstructive sleep apnea. Am J Respir Crit Care Med 2003; 167:15-9.
37 - Larrosa F, Hernandez L, Morello A, Ballester, Quinto L, Montserrat JM. Laser-assisted uvulopalatoplasty for snoring: does it meet the expectations?. Eur Respir J 2004; 24:6670 .

38 - Littner M, Kushida CA, Hartse K, Anderson WM, Davila D, Johnson SF, Wise MS, Hirshkowitz M, Woodson BT. Practice parameters for the use of laser-assisted uvulopalatoplsty: an update for 2000. Sleep 2001; 24:603-19.

39 - Sundaram S, Bridgman S, Lim J, Lassersen T, Sundaram S. Surgery for obstrutive sleep apnea Cochrane database. Syst Rev 2005; 19:CD001004.

40 - Finkelstein Y, Shapiro-Feinberg M, Stein G, Ophir D. Uvulopalatopharyngoplasty vs laser-assisted uvulopalatoplasty: anatomic considerations. Arch Otolaryngol Head Neck Surg 1997; 123:265-76

41 - Sher AE. Obstructive sleep apnea syndrome: a complex disorder of the upper airway. Otolaryngol Clin North Am 1990; 23:593-608 .

42 - Barthel SW, Strome M. Snoring, obstructive sleep apnea and surgery. Med Clin North Am 1999; 83:85-96.

43 - Katsantonis GP, Schweitzer PK, Branham GH, Chambers G Walsh JK. Management of obstructive sleep apnea: comparison of various treatment modalities. Laryngoscope 1988; 98:304-9.

44 - Chervin RD, Theut S, Basseti C, Aldrich MS. Compliance with nasal CPAP can be improved by simples interventions. Sleep 1997; 20:284-9.

45 - Deneke HJ, Meyer R. Plastic Surgery of the head and neck: rhinoplasty. New York: Springer-Verlag; 1967.

46 - Converse JM, ed. Reconstructive plastic surgery. Philadelphia: WB Saunders;1977.

47 - Sampaio PL, Galindo C, Voegels RL. Tratamento cirúrgico das conchas nasais. In: Tratado de otorrinolaringologia. Volume V. Editora Revinter, São Paulo, 2002.

48 - Maw J, Marsan J. Uvulopalatopharyngoplasty versus laserassisted uvulopalatopharyngoplasty in the treatemeant of snoring. J Otolaryngol 1997; 26:232-5.

49 - Miljeteic $\mathrm{H}$. UPPGH in the treatemeant of the obstructive sleep apnea. Acta Otolaryngol 1992; 492:86-9.

50 - Powell NB, Riley RW, Troell RJ, Blumen MB, Guilleminault C. Radiofrequency volumetric reduction of the tongue A porcine pilot study for the treatment of obstructive sleap apnea syndrome. Chest 1997; 111:1348-55. 